\title{
A nonlinear modification for improving dynamic anti-windup compensation
}

\author{
Matthew C. Turner ${ }^{\mathrm{a}}$, Murray Kerr ${ }^{\mathrm{b}}$ \\ ${ }^{a}$ Department of Engineering, University of Leicester, Leicester, LE1 7RH, UK. \\ ${ }^{\mathrm{b}}$ Deimos Space SL, Madrid, 28760, Spain
}

\begin{abstract}
This paper presents a simple modification which can be made to a pre-designed dynamic anti-windup scheme in order to improve its performance. Roughly speaking, the modification enables the dynamic anti-windup compensator to act more like a static anti-windup compensator in certain circumstances. In particular, the modification enables the output of the compensator to decay more quickly than if it were absent, thereby effecting a swifter recovery of linear behaviour. The modification is therefore suitable for - and indeed motivated by - applications where the original anti-windup compensator contains slow poles, resulting in a potentially lengthy recovery of linear behaviour. The paper describes in detail the modification and presents conditions under which it is able to preserve stability.
\end{abstract}

Key words: saturation, anti-windup, sector-bounds

\section{Introduction}

Anti-windup compensators supplement baseline control systems in order to improve their performance during control signal saturation. The idea of anti-windup originated many years ago but the concept has been re-examined extensively by the research community over the last two decades and many new techniques for anti-windup analysis and design have been proposed - see [1-6] for instance.

Anti-windup compensators are activated upon saturation of the control signal $u(t)$, normally by observing the signal $\tilde{u}(t):=u(t)-\operatorname{sat}(u(t))=: \mathrm{Dz}(u(t))$ : once this signal is non-zero the anti-windup compensator becomes active and, if designed properly, should maintain stability and improve performance during periods of saturation. A linear static anti-windup compensator has no dynamics and thus, once

Email addresses: mct 6@le.ac.uk (Matthew C. Turner), murray.kerr@deimos-space.com (Murray Kerr). 
saturation is over $(\tilde{u}(t)=0$ for $t>T)$, the output of the anti-windup compensator, $v(t)$ will also be zero for all $t>T$. A linear dynamic anti-windup compensator behaves differently: once saturation is over, and again $\tilde{u}(t)=0$ for $t>T$, its states decay exponentially to zero i.e. $\left\|x_{a w}(t)\right\| \leq \alpha_{1} \exp \left[-\alpha_{2}(t-T)\right]\left\|x_{a w}(T)\right\|$ for some $\alpha_{1}, \alpha_{2}>0$. Thus, if the anti-windup compensator contains slow modes, its output may take considerable time to decay to negligible values, thereby prolonging the time taken for the system to recover from the effects of saturation.

Modern anti-windup compensators are typically designed using variants of the Multivariable Circle Criterion; that is they are designed using quadratic Lyapunov functions with the aid of sector bounds on the saturation/deadzone nonlinearities. This approach essentially requires a certain transfer function to be strictly positive real. In order for the anti-windup compensator to bestow strict positive realness (and therefore stabilise the system during periods of saturation), it may be necessary for the compensator to contain slow modes. The price paid for stability, therefore, may be a slow recovery of linear behaviour after saturation has occurred: this is contrary to the swift recovery of linear behaviour traditionally sought [7].

This paper proposes a nonlinear modification which can be retro-fitted to a predesigned (dynamic) anti-windup compensator (a retro-fit to a retro-fit) to enable the output of the anti-windup compensator to decay more rapidly after saturation has ceased. The nonlinear modification operates by monitoring the level of saturation, and attenuating the magnitude of the anti-windup compensator's output depending on this: the nonlinear modification can be considered as a nonlinear gain. It transpires that the use of this nonlinear gain, together with a simple filter of order equal to the number of anti-windup outputs, can enable stability to be maintained whilst also improving small signal performance. These ideas were motivated by a flight control application considered by the authors $([8,9]$ and in unpublished work) which suffered a similar issue to that described above. Although the solution to these problems was a little different in the motivating work, some key ideas remain the same in this paper.

It is stressed that this paper considers a nonlinear modification to a pre-designed anti-windup compensator. It does not consider the design of an a priori nonlinear compensator ([10-16]). The latter type of compensator may perform better, and perhaps even may be necessary in some cases ([17]), but typically the design conditions are more complex and the dynamics may be faster and therefore more difficult to implement in practice. The advantage of the modification proposed here is that it is simple to both design and implement and therefore in keeping with the ethos of anti-windup compensation.

Notation. Notation is standard throughout. The saturation function is defined as

$$
\operatorname{sat}(u)=\operatorname{sign}(u) \min \{\bar{u},|u|\} \quad \bar{u}>0
$$

The deadzone function is defined as

$$
\operatorname{Dz}(u)=\operatorname{sign}(u) \max \{0,|u|-\bar{u}\} \quad \bar{u}>0
$$


The identity sat $(u)=u-\operatorname{Dz}(u)$ holds. Frequently, the notation $\tilde{u}=\mathrm{Dz}(u)$ is used.

The identity matrix of size $m$ is denoted $I_{m}$. A vector of size $m$ whose elements are all unity is defined as $\mathbf{1}_{m}$. A positive (negative) definite symmetric matrix $M$ is indicated by $M>(<) 0$.

\section{System Architecture}

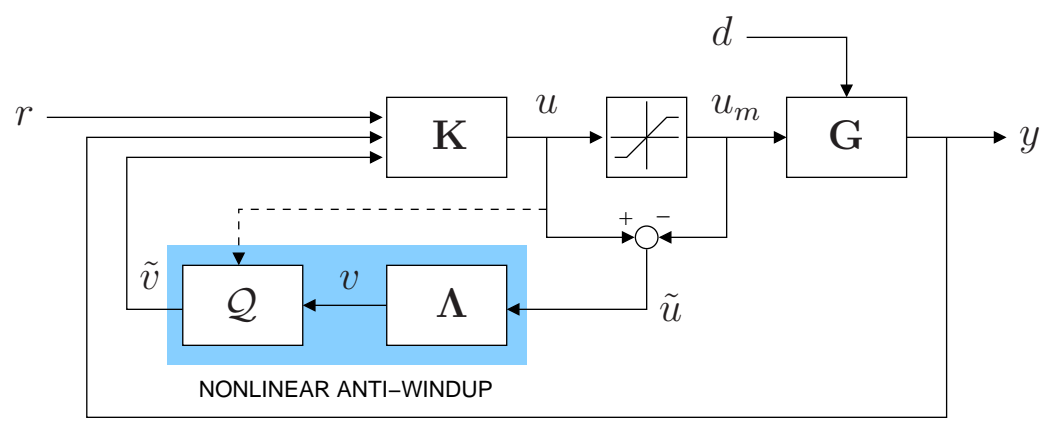

Fig. 1. Anti-windup configuration. In the standard anti-windup configuration $\mathcal{Q}$ is the identity; in this paper, $\mathcal{Q}$ is a nonlinear operator.

The paper considers the system depicted in Figure 1 , where $y \in \mathbb{R}^{p}$ represents the plant measurements; $u \in \mathbb{R}$ and $u_{m}=\operatorname{sat}(u)$ the controller output and the plant input respectively; $r \in \mathbb{R}^{n_{r}}$ the reference demand, typically with $n_{r}=1$; and $d \in \mathbb{R}^{n_{d}}$ the disturbance. The signal which activates the anti-windup compensator is $\operatorname{Dz}(u)=: \tilde{u} \in \mathbb{R}$ and $\tilde{v} \in \mathbb{R}^{n_{v}}$ is the signal the anti-windup compensator injects into the controller. The linear dynamics of the plant and the controller are given by the state-space realisations:

$$
\begin{gathered}
\mathbf{G} \sim\left\{\begin{array}{c}
\dot{x}_{p}=A_{p} x_{p}+B_{p} u_{m}+B_{p d} d \\
y=C_{p} x_{p}+D_{p d} d
\end{array}\right. \\
\mathbf{K} \sim\left\{\begin{array}{c}
\dot{x}_{c}=A_{c} x_{c}+B_{c} y+B_{c r} r+B_{c v} \tilde{v} \\
u=C_{c} x_{c}+D_{c} y+D_{c r} r+D_{c v} \tilde{v}
\end{array}\right.
\end{gathered}
$$

The state dimensions of the plant and the controller are, respectively, $n_{p}$ and $n_{c}$. It is assumed throughout that $A_{p}$ is Hurwitz and that, in the absence of saturation, $\mathbf{K}$ internally stabilises $\mathbf{G}$.

The nonlinear anti-windup compensator, depicted in Figure 1, is a cascade of two components. The first is a linear component, which is assumed to be pre-designed and, for simplicity, strictly proper. This has state-space realisation:

$$
\Lambda \sim\left\{\begin{array}{l}
\dot{x}_{a}=A_{a} x_{a}+B_{a} \tilde{u} \\
v=C_{a} x_{a}
\end{array}\right.
$$


The second component is the anti-windup modification filter which is a dynamic nonlinear operator with state-space realisation

$$
\mathcal{Q} \sim\left\{\begin{array}{r}
\dot{x}_{q}=f_{q}\left(x_{q}, u, v\right) \\
\tilde{v}=g_{q}\left(x_{q}, u, v\right)
\end{array}\right.
$$

The functions $f_{q}(., .,$.$) and g_{q}(., .,$.$) will be specified later, as will the state-vector$ dimension. If $\mathcal{Q}$ is the identity operator, standard linear anti-windup compensation is recovered, i.e. $\tilde{v}=v$. In this case, providing $\Lambda$ has been designed to globally stabilise the origin of the system, nothing else needs proving. This paper considers a $\mathcal{Q}$, not equal to the identity, which improves performance in some sense.

\subsection{Description of the modification}

Before providing the technical details behind the nonlinear modification filter, $\mathcal{Q}$, it is useful to describe the way in which it is envisaged to operate. The rationale behind the introduction of the nonlinear modification is, as described in the previous section, to provide faster attenuation of the anti-windup compensation signal after saturation has ceased, so that the return to linear behaviour is swifter. However, when significant saturation takes place $(u>>\bar{u})$, it is desirable to leave in place the anti-windup compensator so that it functions as originally intended. From a simplistic perspective, the purpose of the nonlinear operator $\mathcal{Q}$ is, then, to adjust (in a nonlinear fashion) the gain of the anti-windup compensator between zero (no saturation) and one (full saturation).

This nonlinear attenuation is accomplished by monitoring the level of saturation, through measurement of $u$ and then using this to set the "gain" of $\mathcal{Q}$. However, it transpires that such a strategy may result in unsatisfactory transient performance (especially when the control signal passes from fully saturated in one direction to fully saturated in the opposite direction) and leads to an implicit expression for the control signal $u$ which may result in the system being ill-posed. For these reasons, $\mathcal{Q}$ features some dynamics and, as will be clear later, the choice of these dynamics effectively reduces to choosing the bandwidth of a linear filter.

\section{Motivating Example}

To illustrate the problem described above, we consider an example which has been inspired by the authors' work on anti-windup design for PIO avoidance [9]. The example is realistic as the essence of the problem is similar to that of the applied work described in [9], but it is also illustrative due to the simple interpretation which can be given to the anti-windup compensator.

In this case, the dynamics of plant comprise the lateral dynamics of an aircraft combined with a first order actuator. The plant output to be controlled is the roll 
attitude, $\phi(t)$, and the controller measures this in addition to the roll rate $p(t)$, the actuator position $x_{a c t}(t)$ and roll attitude demand $\phi_{d}(t)$ i.e. $r=\phi_{d}$. The controller is purely static and hence, when $\tilde{v}(t) \equiv v(t)$ (no modification), can be described via:

$$
u(t)=D_{c} y(t)+D_{c r} r(t)+D_{c v} v(t)
$$

For this system, an anti-windup compensator was designed using the method of [18] and this yielded a compensator with output $v=\left[\begin{array}{ll}v_{1}^{\prime} & v_{2}^{\prime}\end{array}\right]^{\prime}, v_{1} \in \mathbb{R}, v_{2} \in \mathbb{R}^{3}$. The corresponding $D_{c v}=\left[\begin{array}{ll}-1 & D_{c r}\end{array}\right]$, so the effect of the anti-windup compensator can be combined into a "total" $v_{T}(t)$

$$
v_{T}(t):=-v_{1}(t)+D_{c r} v_{2}(t) \in \mathbb{R}
$$

It is simpler to observe the anti-windup action from this signal than observe the four signals associated with $v(t)$.

Ideally, we would like $v_{T}(t)$ to be inactive apart from when saturation occurs. However, in the case of dynamic anti-windup compensators, if the dynamics are sufficiently slow, $v_{T}$ will linger after saturation has ceased. This is because, assuming $|u(t)|<\bar{u} \quad \forall t>t_{s}$, we have

$$
v_{T}(t)=C_{a w} \exp \left[A_{a w}\left(t-t_{s}\right)\right] x_{a w}\left(t_{s}\right)
$$

Unfortunately, due to the mix of slow and fast dynamics in the plant, the antiwindup compensator designed using the method in [18] resulted in a slow real pole at $\approx 10^{-3}$ radian/s. This meant that recovery of linear behaviour was slow.

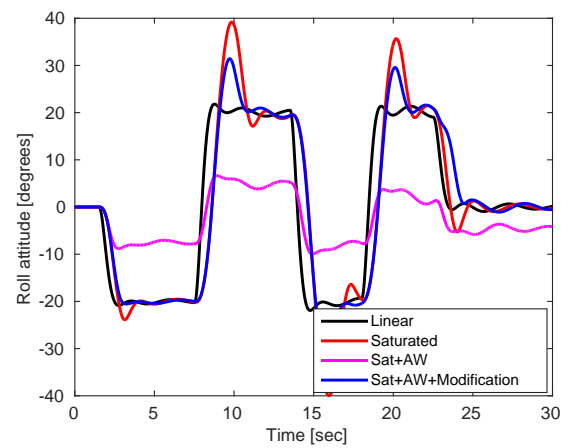

(a) Roll Response

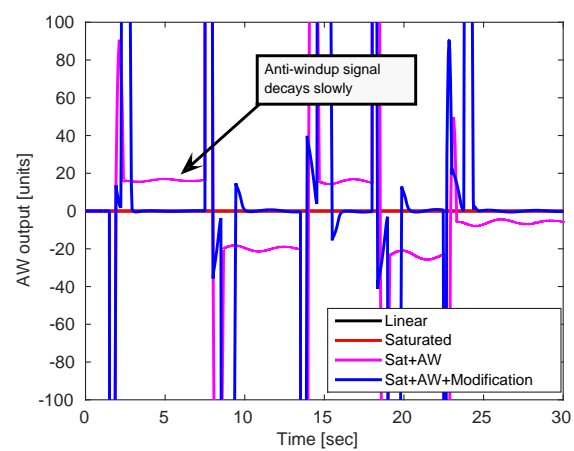

(b) Anti-windup signal, $v_{T}(t)$

Fig. 2. Sanitised aircraft example response. Note that the anti-windup signals $v_{T}(t)$ are not present in the linear and saturated cases in (b)

Figure 2 (a) shows the roll attitude response of the aircraft example under various conditions. The saturation limits were 25 units and the reference demand, $\phi_{d}(t)$ is a series of pulses. The linear response (black) is well-damped and brisk, but when saturation (red) is introduced - and no anti-windup is used - the response contains large overshoots. For certain reference demands, the system enters a limit cycle. Unfortunately, when full-order anti-windup above is introduced (magenta), the response does not improve much: although the overshoots lessen, the full-order anti-windup 
compensator's action is too aggressive and (see Figure 2 (b)) the anti-windup signal associated with this compensator decays very slowly, meaning that the system fails to track the pulse references (Figure 2 (a)). To prevent these tracking problems, the modification described earlier is introduced to the system. The blue trace in Figure 2 (a) shows that tracking performance is now much improved; this can be explained by the faster decay of the anti-windup signal, $v_{T}(t)$ which is seen in Figure 2 (b). The following sections describe how such a nonlinear modification can be added without compromising stability.

\section{Problem Formulation}

\subsection{Nonlinear Modification Filter $\mathcal{Q}$}

Before formally stating the problem, the dynamics of the nonlinear modification filter, $\mathcal{Q}$, are defined and the dynamics of the system are re-written. Firstly, the dynamics of $\mathcal{Q}$ are considered to take the following form:

$$
\mathcal{Q} \sim\left\{\begin{array}{l}
\dot{x}_{q}=A_{q} x_{q}+(1-\beta(u)) B_{q} v \\
\tilde{v}=-C_{q} x_{q}+v
\end{array}\right.
$$

where $\beta():. \mathbb{R} \mapsto[0,1]$ is a nonlinear function defined by

$$
\beta(u):=\operatorname{Dz}(u) / u=\max \{0,(|u|-\bar{u}) /|u|\}
$$

Note that $\beta(u)=0$ when $|u|<\bar{u}$ i.e. when saturation does not take place. The state-space matrices $\left(A_{q}, B_{q}, C_{q}\right)$ are not specified at the moment, but if they are chosen to correspond to those of a low-pass filter i.e.

$$
\left(A_{q}, B_{q}, C_{q}\right)=\left(-\lambda I_{n_{v}}, I_{n_{v}}, \lambda I_{n_{v}}\right) \quad \lambda>0
$$

it follows that when saturation is not active $(\beta(u)=0)$ the output $\tilde{v} \rightarrow 0$ i.e. the output of $\mathcal{Q}$ decays to zero. Similarly if saturation is present and extensive, that is $|u|>>\bar{u}$, then $\beta(u) \rightarrow 1$ which implies that $x_{q} \rightarrow 0$ and thus the output $\tilde{v} \rightarrow v$. This form of $\mathcal{Q}$ functions in the way envisaged in Section 2.1, although it is not the only choice possible.

\subsection{LFT modelling}

The dynamics of the plant and the controller can be combined to obtain

$$
\mathbf{P}_{c l} \sim\left\{\begin{aligned}
\dot{x}_{c l} & =A_{c l} x_{c l}+B_{c l u} \tilde{u}+B_{c l v} \tilde{v} \\
u & =C_{c l} x_{c l}+D_{c l v} \tilde{v}
\end{aligned}\right.
$$




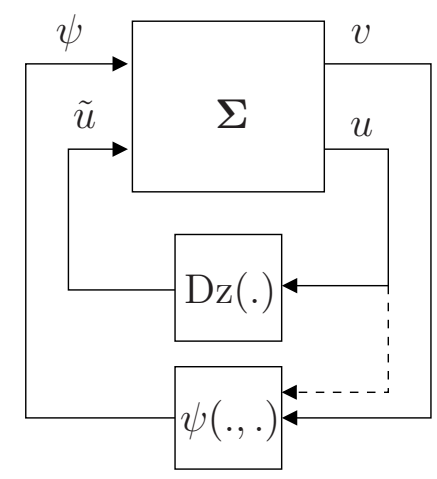

Fig. 3. Equivalent Representation of Nonlinear Anti-windup using an "LFT"

where expressions for the above matrices can be found in the appendix. The matrix $A_{c l}$ is Hurwitz because it is assumed that the nominal controller stabilises the system without saturation. For convenience, the nonlinear function $\psi(.,):. \mathbb{R}^{n_{v}} \times \mathbb{R} \mapsto$ $\mathbb{R}^{n_{v}}$ is defined:

$$
\psi(v, u)=\beta(u) v
$$

Thus the dynamics of the plant and the controller, (12), the linear anti-windup compensator (5), and the modification filter (10), can be combined in the following "LFT" form, illustrated in Figure 3, where the LTI element $\Sigma$ has state-space realisation:

$$
\Sigma \sim\left\{\begin{array}{l}
\dot{x}=A x+B_{1} \tilde{u}+B_{2} \psi(v, u) \\
u=C_{1} x \\
v=C_{2} x
\end{array}\right.
$$

The state-space matrices are given in the appendix.

It is assumed that an anti-windup compensator $\Lambda$ has been designed such that the closed loop system, with $\mathcal{Q}$ replaced by the identity, is asymptotically stable. Moreover, because many popular anti-windup design techniques are, in essence, based on an application of the Circle Criterion, it is assumed that the system without the dynamics of $\mathcal{Q}$, satisfies the matrix inequality associated with the Circle Criterion. When $\mathcal{Q}$ is omitted, the dynamics of the system become

$$
\Sigma_{\ell} \sim\left\{\begin{aligned}
\dot{x}_{\ell} & =A_{\ell} x_{\ell}+B_{\ell} \tilde{u} \\
u & =C_{\ell} x
\end{aligned}\right.
$$

where the state-space matrices $\left(A_{\ell}, B_{\ell}, C_{\ell}\right)$ are defined in the appendix. This motivates the main assumption of the paper

Assumption 1 There exists a symmetric positive definite matrix $P_{\ell}>0$ and a positive scalar $w_{\ell}>0$ such that the following matrix inequality is satisfied:

$$
\left[\begin{array}{cc}
A_{\ell}^{\prime} P_{\ell}+P_{\ell} A_{\ell} P_{\ell} B_{\ell}+C_{\ell}^{\prime} w_{\ell} \\
\star & -2 w_{\ell}
\end{array}\right]<0
$$


Remark 1: This paper considers asymptotically stable open-loop systems; $A_{p}$ is a Hurwitz matrix. In this case, there will always exist a full-order $\left(n_{a}=n_{p}\right)$ antiwindup compensator of the form (5) which renders the saturated system exponentially stable via the Circle Criterion. However, Assumption 1 does not stipulate that the compensator must be full-order and often a low-order compensator is perfectly satisfactory $([19,20])$. In fact, Assumption 1 is not necessary: all that is really required is for the saturated system with anti-windup to be asymptotically stable by an appropriate Lyapunov function. However, Assumption 1 would be typical for many modern anti-windup designs and allows neat results to be obtained.

Remark 2: Assumption 1 implies that $A_{c l}$ is Hurwitz, that is the linear controller stabilises the linear plant. This is evident from the structure of $A_{\ell}$ in the appendix.

The following is the problem addressed in the remainder of the paper.

Problem 2 Consider the system (14) where $\tilde{u}$ and $\psi(v, u)$ are defined in equations (2) and (13). Find conditions, in the form of linear matrix inequalities, which ensure that the origin of the system is globally asymptotically stable.

Remark 3: Problem 2 is focused on ensuring the origin of the system with antiwindup modification is asymptotically stable; it does not explicitly consider performance, which is the reason for introducing the modification filter. One can intuitively understand the reason why the modification filter might yield improved transient behaviour (as explained in Section 3) but characterising this mathematically is difficult. Firstly, the modification filter is not expected to improve the $\mathcal{L}_{2}$ gain properties of the anti-windup compensator because, for large levels of saturation (i.e. "large" $\mathcal{L}_{2}$ signals) the modification filter essentially makes no modification. Similarly, a decay rate charaterisation seems to fail because, the decay rate of the modified compensator $\mathcal{Q}$ and $\Lambda$ is always determined by the slowest element (i.e. $\Lambda)$. However, the intuitive behaviour of the compensator, together with its stability guarantees should be sufficient for improved time-domain performance to be observed.

\section{Main Results}

\subsection{Sector Conditions}

One of the nonlinearities featured in $\Sigma$ is the deadzone nonlinearity, $\tilde{u}=\mathrm{Dz}(u)$ which is well known to satisfy certain sector inequalities. The purpose of this subsection is to derive similar sector conditions for the nonlinear element $\psi(v, u)$ and also - taking inspiration from [21] - combinations of the nonlinearities $\mathrm{Dz}(u)$ and 
$\psi(v, u)$. These conditions are more palatable, with the shorthand

$$
\psi_{i}:=\psi_{i}(v, u)=\beta(u) v_{i}
$$

The following result can be stated.

Lemma 3 Consider the nonlinear functions $\mathrm{Dz}():. \mathbb{R} \mapsto \mathbb{R}$ and $\psi(.,):. \mathbb{R}^{n_{v}} \times$ $\mathbb{R} \mapsto \mathbb{R}^{n_{v}}$, as defined in equations (2) and (13), and let $\psi_{i}$ be defined as in equation (17). Then the following inequalities hold for all positive scalars $\eta$ :

$$
\begin{aligned}
\operatorname{Dz}(u) \eta(u-\operatorname{Dz}(u)) \geq 0 & \forall u \in \mathbb{R} & \\
\psi_{i} \eta\left(v_{i}-\psi_{i}\right) \geq 0 & \forall u, v_{i} \in \mathbb{R} & \forall i \in\left\{1, \ldots, n_{v}\right\} \\
\left(\mathrm{Dz}(u)+\psi_{i}\right) \eta\left[u+v_{i}-\left(\mathrm{Dz}(u)+\psi_{i}\right)\right] \geq 0 & \forall u, v_{i} \in \mathbb{R} & \forall i \in\left\{1, \ldots, n_{v}\right\} \\
\left(\mathrm{Dz}(u)-\psi_{i}\right) \eta\left[u-v_{i}-\left(\mathrm{Dz}(u)-\psi_{i}\right)\right] \geq 0 & \forall u, v_{i} \in \mathbb{R} & \forall i \in\left\{1, \ldots, n_{v}\right\}
\end{aligned}
$$

\section{Proof:}

i) Inequality (18) is simply the standard sector inequality.

ii) Inequality (19). The left hand side may be written as

$$
\begin{aligned}
\psi_{i} \eta\left(v_{i}-\psi_{i}\right) & =v_{i} \beta(u) \eta(1-\beta(u)) v_{i} \\
& =v_{i}^{2} \eta \beta(u)(1-\beta(u)) \geq 0
\end{aligned}
$$

where the last inequality follows because $\beta(u) \in[0,1]$.

iii) Inequality (20). Noting that $\mathrm{Dz}(u)=\beta(u) u$, we can write:

$$
\begin{aligned}
\left(\mathrm{Dz}(u)+\psi_{i}\right) \eta\left[u+v_{i}-\left(\mathrm{Dz}(u)+\psi_{i}\right)\right] & =\left(u+v_{i}\right) \beta(u) \eta(1-\beta(u))\left(u+v_{i}\right) \\
& =\left(u+v_{i}\right)^{2} \eta \beta(u)(1-\beta(u))
\end{aligned}
$$

where again the last inequality follows because $\beta(u) \in[0,1]$.

iv) This follows by similar reasoning to the third.

\subsection{Stability conditions}

Lemma 3 can be used in the derivation of Lyapunov conditions for stability using a Lur'e-type Lyapunov function.

Proposition 4 Consider the system described by equation (14). Then the origin is globally asymptotically stable if there exist a positive definite symmetric matrix $P>0$, a diagonal positive definite matrix $W_{2}>0$, positive scalars $w_{1}>0$ and $\eta>$ 
0 , and diagonal matrices $X_{1}, X_{2}$ which satisfy the following matrix inequalities

$$
\begin{aligned}
& {\left[\begin{array}{ccc}
A^{\prime} P+P A & P B_{1}+C_{1}^{\prime}\left(w_{1}+\mathbf{1}^{\prime} X_{1} \mathbf{1}\right)+C_{2}^{\prime} X_{2} \mathbf{1}+A^{\prime} C_{1}^{\prime} \eta & P B_{2}+C_{2}^{\prime}\left(W_{2}+X_{1}\right)+C_{1}^{\prime} \mathbf{1}^{\prime} X_{2} \\
\star & -2 w_{1}-2 \mathbf{1}^{\prime} X_{1} \mathbf{1}+\eta\left(C_{1} B_{1} B_{1}^{\prime} C_{1}^{\prime}\right) & -2 \mathbf{1}^{\prime} X_{2}+\eta C_{1} B_{2} \\
\star & \star & -2 W_{2}-2 X_{1}
\end{array}\right]<0} \\
& X_{1}+X_{2}>0 \\
& X_{1}-X_{2}>0
\end{aligned}
$$

Proof: The proof of the proposition is similar to classical absolute stability proofs [22]. First we choose a Lyapunov function

$$
V(x)=x^{\prime} P x+2 \eta \int_{0}^{C_{1} x} \operatorname{Dz}(\sigma) d \sigma
$$

where $P>0$ is a positive define matrix and $\eta>0$ a positive scalar. The derivative along the trajectories of the system (14) can be calculated as

$$
\begin{aligned}
\dot{V}(x) & =2\left(x^{\prime} P+\eta \mathrm{Dz}(u) C_{1}\right)\left(A x+B_{1} \tilde{u}+B_{2} \psi\right) \\
& =\left[\begin{array}{c}
x \\
\tilde{u} \\
\psi
\end{array}\right]^{\prime}\left[\begin{array}{ccc}
A^{\prime} P+P A & P B_{1}+A^{\prime} C_{1}^{\prime} \eta & P B_{2} \\
\star & \eta\left(C_{1} B_{1}+B_{1}^{\prime} C_{1}^{\prime}\right) & \eta C_{1} B_{2} \\
\star & \star & 0
\end{array}\right]\left[\begin{array}{l}
x \\
\tilde{u} \\
\psi
\end{array}\right]
\end{aligned}
$$

Using the state-space realisation of $\Sigma$ in (14) we can re-write the sector conditions in Lemma 3 as:

Condition (i) (from inequality (18)):

$$
\tilde{u}^{\prime} w_{1}\left(C_{1} x-\tilde{u}\right) \geq 0 \quad w_{1}>0
$$

Condition (ii) (from inequality (19)):

$$
\psi^{\prime} W_{2}\left(C_{2} x-\psi\right) \geq 0 \quad W_{2}=\operatorname{diag}\left(w_{21}, \ldots, w_{2 m}\right)>0
$$

Condition (iii): Using inequality (20), for any positive scalars $w_{3, i}$, it follows that

$$
2 \sum_{i}^{n_{v}}\left(\operatorname{Dz}(u)-\psi_{i}\right) w_{3, i}\left[u-v_{i}-\left(\operatorname{Dz}(u)-\psi_{i}\right)\right] \geq 0
$$

Defining

$$
W_{3}=\operatorname{diag}\left(w_{31}, \ldots, w_{3 m}\right)>0
$$


and noting that

$$
\begin{aligned}
\mathbf{1}^{\prime} W_{3} \mathbf{1} & =\sum_{i}^{n_{v}} w_{3, i} \\
\mathbf{1}^{\prime} W_{3} & =\left[\begin{array}{llll}
w_{3,1} & w_{3,2} & \cdots & w_{3, n_{v}}
\end{array}\right]
\end{aligned}
$$

allows inequality (34) to be written as

$$
\begin{aligned}
& {\left[\begin{array}{l}
x \\
\tilde{u} \\
\psi
\end{array}\right]^{\prime}\left[\begin{array}{ccc}
0 & C_{1}^{\prime} \mathbf{1}^{\prime} W_{3} \mathbf{1}+C_{2}^{\prime} W_{3} \mathbf{1} & C_{1}^{\prime} \mathbf{1}^{\prime} W_{3}+C_{2}^{\prime} W_{3} \\
\star & -2 \mathbf{1}^{\prime} W_{3} \mathbf{1} & -2 \mathbf{1}^{\prime} W_{3} \\
\star & \star & -2 W_{3}
\end{array}\right]\left[\begin{array}{l}
x \\
\tilde{u} \\
\psi
\end{array}\right] \geq 0} \\
& W_{3}=\operatorname{diag}\left(w_{31}, \ldots, w_{3 m}\right)>0
\end{aligned}
$$

Condition (iv): This follows in a similar manner to inequality (38), except that the starting point is inequality (21).

$$
\begin{aligned}
& {\left[\begin{array}{l}
x \\
\tilde{u} \\
\psi
\end{array}\right]^{\prime}\left[\begin{array}{ccc}
0 & C_{1}^{\prime} \mathbf{1}^{\prime} W_{4} \mathbf{1}-C_{2}^{\prime} W_{4} \mathbf{1} & -C_{1}^{\prime} \mathbf{1}^{\prime} W_{4}+C_{2}^{\prime} W_{4} \\
\star & -2 \mathbf{1}^{\prime} W_{4} \mathbf{1} & 2 \mathbf{1}^{\prime} W_{4} \\
\star & \star & -2 W_{4}
\end{array}\right]\left[\begin{array}{l}
x \\
\tilde{u} \\
\psi
\end{array}\right] \geq 0} \\
& W_{4}=\operatorname{diag}\left(w_{41}, \ldots, w_{4 m}\right)>0
\end{aligned}
$$

Appending inequalities (32)-(40) to the derivative of our Lyapunov function (31) yields the inequality

$$
\dot{V}(x) \leq\left[\begin{array}{c}
x \\
\tilde{u} \\
\psi
\end{array}\right]^{\prime}\left[\begin{array}{cc}
A^{\prime} P+P A P B_{1}+C_{1}^{\prime}\left(w_{1}+\mathbf{1}^{\prime}\left(W_{3}+W_{4}\right) \mathbf{1}\right)+C_{2}^{\prime}\left(W_{3}-W_{4}\right) \mathbf{1}+A^{\prime} C_{1}^{\prime} \eta \\
\star & -2 w_{1}-2 \mathbf{1}^{\prime}\left(W_{3}+W_{4}\right) \mathbf{1}+\eta\left(C_{1} B_{1}+B_{1}^{\prime} C_{1}^{\prime}\right) \\
\star & \\
& P B_{2}+C_{2}^{\prime}\left(W_{2}+W_{3}+W_{4}\right)+C_{1}^{\prime} \mathbf{1}^{\prime}\left(W_{3}-W_{4}\right) \\
& -2 \mathbf{1}^{\prime}\left(W_{3}-W_{4}\right)+\eta C_{1} B_{2} \\
-2 W_{2}-2\left(W_{3}+W_{4}\right)
\end{array}\right]\left[\begin{array}{c}
x \\
\tilde{u} \\
\psi
\end{array}\right]
$$

Defining

$$
\begin{aligned}
& X_{1}:=W_{3}+W_{4} \\
& X_{2}:=W_{3}-W_{4}
\end{aligned}
$$


gives the inequality (26). This means that $W_{3}$ and $W_{4}$ can be recovered as

$$
\begin{aligned}
& W_{3}=\frac{1}{2}\left(X_{1}+X_{2}\right) \\
& W_{4}=\frac{1}{2}\left(X_{1}-X_{2}\right)
\end{aligned}
$$

Inequalities (27) and (28) ensure $W_{3}$ and $W_{4}$ are positive definite and diagonal.

Proposition 4 gives conditions which ensure that the system with anti-windup and the modification filter $\mathcal{Q}$ are asymptotically stable. It assumes that the plant, the controller and the filter dynamics are given. A typical form of filter was argued to be a bank of first order filters described by equation (4.1). It is next shown that, if Assumption 1 is satisfied, there will always exist an anti-windup modification filter $\mathcal{Q}$ ensuring stability.

Proposition 5 Assume the plant, controller and anti-windup compensator dynamics are given and let Assumption 1 be satisfied. Then there always exist matrices $\left(A_{q}, B_{q}, C_{q}\right)$ such that Proposition 4 is satisfied.

Proof: Let $X_{1}$ and $X_{2}$ be two sufficiently "small" matrices satisfying inequalities (27) and (28). Also, let $\eta$ be a sufficiently small scalar. In this case, a sufficient condition for inequality (26) to be satisfied is for the following inequality to hold:

$$
\left[\begin{array}{ccc}
A^{\prime} P+P A & P B_{1}+C_{1}^{\prime} w_{1} & P B_{2}+C_{2}^{\prime} W_{2} \\
\star & -2 w_{1} & 0 \\
\star & \star & -2 W_{2}
\end{array}\right]<0
$$

The matrix $B_{2}$ is given by (see appendix)

$$
B_{2}=\left[\begin{array}{c}
0 \\
0 \\
-B_{q}
\end{array}\right]
$$

Thus, for sufficiently small $B_{q}$, and sufficiently small $W_{2}$, it follows that inequality (47) will be satisfied if their exist matrices $P>0$ and a scalar $w_{1}>0$ such that following inequality is satisfied:

$$
\left[\begin{array}{cc}
A^{\prime} P+P A & P B_{1}+C_{1}^{\prime} w_{1} \\
\star & -2 w_{1}
\end{array}\right]<0
$$


Referring to the appendix, it can be seen that this matrix inequality has the structure

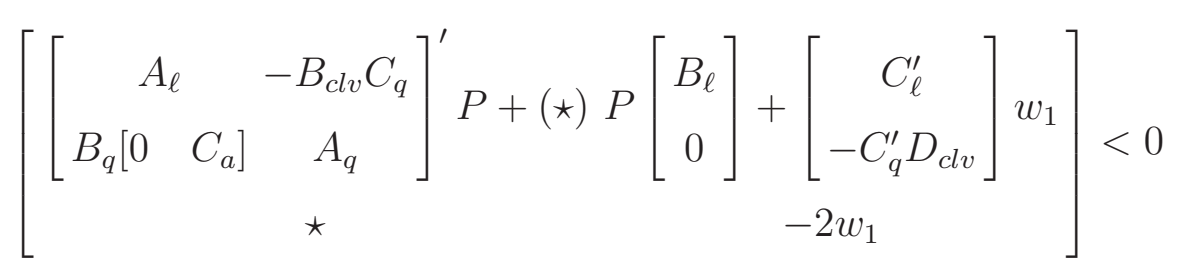

Let $\tilde{A}_{q} \in \mathbb{R}^{n_{q}}$ be a Hurwitz matrix; then there exists a $P_{q}>0$ satisfying

$$
\tilde{A}_{q}^{\prime} P_{q}+P_{q} \tilde{A}_{q}<0
$$

Now let $A_{q}=\epsilon \tilde{A}_{q}$ and let $P=\operatorname{blockdiag}\left(P_{\ell}, P_{q}\right), P_{\ell}>0$. Then inequality (50) will be satisfied for sufficiently small $\epsilon>0$ and $B_{q}$, or sufficiently small $\left(B_{q}, C_{q}\right)$, if the following inequality is satisfied for some matrix $P_{\ell}>0$ and scalar $w_{1}>0$

$$
\left[\begin{array}{cc}
A_{\ell}^{\prime} P_{\ell}+P_{\ell} A_{\ell} & P_{\ell} B_{\ell}+C_{\ell}^{\prime} w_{1} \\
\star & -2 w_{1}
\end{array}\right]<0
$$

which is exactly Assumption 1.

\subsection{Improved stability conditions}

The results of the previous subject exploited sector boundedness of the deadzone nonlinearity, along with the three new sector conditions introduced in Lemma 3 regarding the nonlinearity $\psi(v, u)$ and the deadzone nonlinearity. However, the deadzone nonlinearity is also slope restricted, with the upper bound on its slope being 1 and the lower bound being zero; With the shorthand $\phi(u)=\mathrm{Dz}(u)$, the following inequality is satisfied

$$
0 \leq \frac{\phi\left(u_{1}\right)-\phi\left(u_{2}\right)}{u_{1}-u_{2}} \leq 1 \quad \forall u_{1} \neq u_{2}
$$

In this case the following inequality is satisfied almost everywhere ([23,24]):

$$
\dot{\phi}(u) \eta(\dot{u}-\dot{\phi}(u)) \geq 0 \quad \forall u \in \mathbb{R} \quad \forall \eta>0
$$

In this case, the following Lyapunov function can be used as the basis for proving stability

$$
V(x)=\left[\begin{array}{l}
x \\
\phi
\end{array}\right]^{\prime}\left[\begin{array}{cc}
X & Y \\
Y^{\prime} & z
\end{array}\right]\left[\begin{array}{l}
x \\
\phi
\end{array}\right]+2 \sum_{i=1}^{4} g_{i}(x)
$$


where

$$
\begin{aligned}
& g_{1}(x)=\mu_{1} \int_{0}^{u} \phi(\sigma) d \sigma \geq 0 \quad \forall u \\
& g_{2}(x)=\mu_{2} \int_{0}^{u}[\sigma-\phi(\sigma)] d \sigma \geq 0 \quad \forall u \\
& g_{3}(x)=\mu_{3} \int_{0}^{u}[1-\partial \phi(\sigma)] \sigma d \sigma \geq 0 \quad \forall u \\
& g_{4}(x)=\mu_{4} \int_{0}^{u} \partial \phi(\sigma)[\sigma-\phi(\sigma)] d \sigma \geq 0 \quad \forall u
\end{aligned}
$$

and $\partial($.$) is the sub-differential operator. A neater Lyapunov function which yields$ similar stability conditions can be obtained using the approach of [25]. The Lyapunov function (53) can be used, together with the sector inequalities from Lemma 3 and the slope inequality (52) to prove the following result.

Proposition 6 Consider the system described by equation (14). Then the origin is globally asymptotically stable if there exist a positive definite symmetric matrix $X>0$, a matrix $Y$, a diagonal positive definite matrix $W_{2}>0$, positive scalars $w_{1}>0, z>0, \eta>0$ and $\mu_{i}>0(i \in\{1, \ldots, 4\})$, and diagonal matrices $X_{1}, X_{2}$ which satisfy the following matrix inequalities

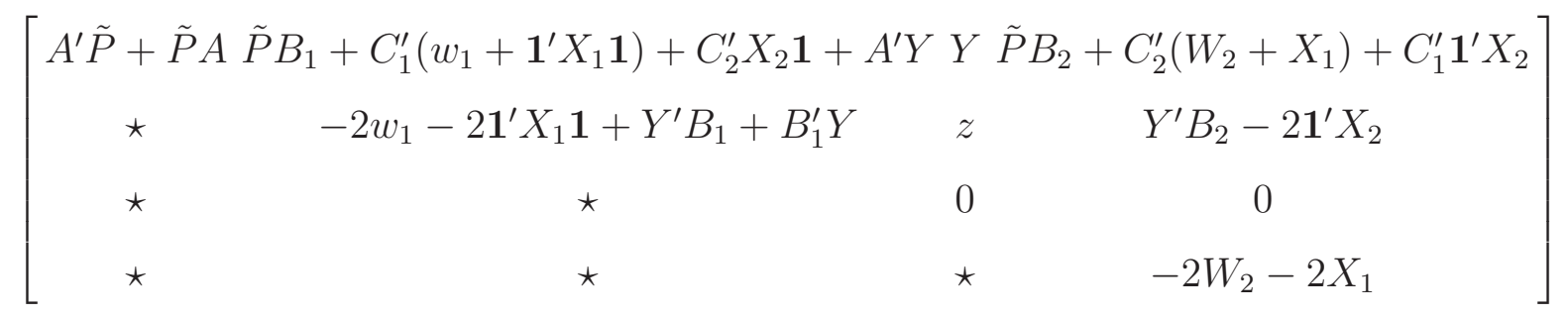

$$
\begin{aligned}
& +\left[\begin{array}{cccc}
0 & \left(\mu_{1}-\mu_{2}\right) A^{\prime} C_{1}^{\prime} & \left(\mu_{4}-\mu_{3}\right) C_{1}^{\prime}+A^{\prime} C_{2}^{\prime} \eta & 0 \\
\star & \left(\mu_{1}-\mu_{2}\right)\left(C_{1} B_{1}+B_{1}^{\prime} C_{1}^{\prime}\right) & -\mu_{4}+B_{1}^{\prime} C_{1}^{\prime} \eta & \left(\mu_{1}-\mu_{2}\right) C_{1} B_{2} \\
\star & \star & -2 \eta & \eta C_{1} B_{2} \\
\star & \star & \star & 0
\end{array}\right]<0 \\
& {\left[\begin{array}{ll}
X & Y \\
\star & z
\end{array}\right]>0} \\
& X_{1}+X_{2}>0 \\
& X_{1}-X_{2}>0
\end{aligned}
$$

Proof: The proof follows in a similar manner to that of Proposition 4, except that the Lyapunov function (53) is used. In addition, the slope restriction (52) is noted to yield:

$$
\dot{\phi}(u) \eta\left(C\left(A x+B_{1} \phi+B_{2} \psi\right)-\dot{\phi}(u)\right) \geq 0
$$


Combining the expression for $\dot{V}$, obtained from (53), and the sector conditions from Lemma 3, after some algebra, yields the result.

\section{Examples}

\subsection{Circuit Example}

This example was used in a number of papers to illustrate the benefits of nonlinear anti-windup [13,21]. The plant and controller have the following state-space matrices:

$$
\begin{aligned}
& {\left[\begin{array}{c|c}
A_{p} & B_{p} \\
\hline C_{p} & D_{p}
\end{array}\right]=\left[\begin{array}{ccc|c}
-10.6000 & -6.0900 & -0.9000 & 1.0000 \\
1.0000 & 0 & 0 & 0 \\
0 & 1.0000 & 0 & 0 \\
\hline-1.0000 & -11.0000 & -30.0000 & 0
\end{array}\right]} \\
& {\left[\begin{array}{c|c}
A_{c} & B_{c} \\
\hline C_{c} & D_{c}
\end{array}\right]=10^{3} \times\left[\begin{array}{cc|c}
-0.0800 & 0 & 0.0010 \\
0.0010 & 0 & 0 \\
\hline \hline 0.0203 & 1.6000 & 0.0800
\end{array}\right]}
\end{aligned}
$$

and $B_{c r}=-B_{c}, D_{c r}=-D_{c}$. The anti-windup terms are injected through

$$
\left[\begin{array}{l}
B_{c v} \\
D_{c v}
\end{array}\right]=\left[\begin{array}{cc}
0 & B_{c} \\
-1 & D_{c}
\end{array}\right]
$$

The anti-windup compensator is a full-order compensator designed using the method described in [18] with $W_{p}=1, W_{r}=0.1$. This anti-windup compensator has an output vector $v \in \mathbb{R}^{2}$ since one output is fed directly to the controller output $\left(v_{1}\right)$ and the other to the controller input $\left(v_{2}\right)$. Therefore, the anti-windup modification filter state-space matrices can be chosen as

$$
\left(A_{q}, B_{q}, C_{q}\right)=\left(-\lambda I_{2}, I_{2}, \lambda I_{2}\right)
$$

where $\lambda>0$. Proposition 5 implies the existence of such a modification filter for sufficiently small $\lambda>0$. For this system, $\lambda$ was chosen as $\lambda=10$ and the matrix inequalities in Proposition 4 were feasible.

Figure 4 shows the plant output and input response of the system for a pulse input demand of magnitude 3 . It can be seen that for this input, the response of the system is sluggish with standard linear anti-windup compensation, but without antiwindup compensation the response is worse. When the anti-windup modification 

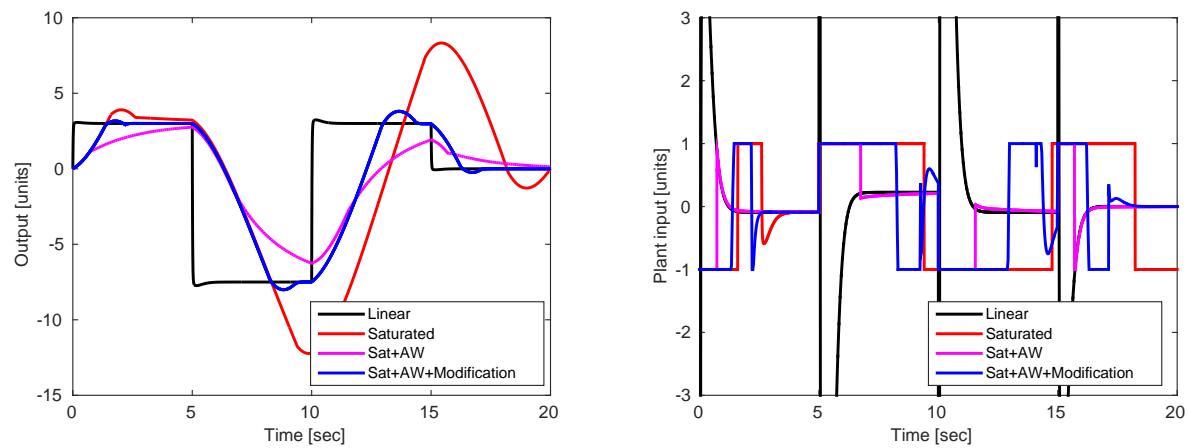

Fig. 4. Circuit example: $y(t)$, large pulse. Nominal linear response: black. Saturated response: red. Standard anti-windup: magenta. Anti-windup with modification filter: solid black

filter is added, the system response retains the briskness of the system without antiwindup compensation, but without the excessive overshoot; clearly the response has improved. The presence of the anti-windup modification filter enables the control signal to be more active, compared to the system without. Improvement is even better for smaller reference demands.

\subsection{Lightly damped example}

This example has similar features to the motivating aircraft example without the complexities present in the real system. The plant has two lightly damped complexconjugate poles close to the imaginary axis. Its state-space matrices are:

$$
\left[\begin{array}{c|c}
A_{p} & B_{p} \\
\hline C_{p} & D_{p}
\end{array}\right]=\left[\begin{array}{cc|c}
0 & 1 & 0 \\
-0.1 & -0.01 & 0.25 \\
\hline 1 & 0 & 0 \\
0 & -1 & 0
\end{array}\right]
$$

The plant has two outputs, with, roughly the second being the derivative of the first. A static controller provides good performance for this system and is given by

$$
D_{c}=\left[\begin{array}{ll}
-8 & -7.2
\end{array}\right], \quad D_{c r}=8, \quad D_{c v}=\left[\begin{array}{ll}
-1 & D_{c}
\end{array}\right]
$$

A full-order anti-windup compensator was designed for this system using the method of [18] with $W_{p}=I_{2}$ and $W_{r}=0.1$. This anti-windup compensator has three inputs to the controller $\left(n_{v}=3\right)$ although, because the controller is static, this actually could be realised as just one input as mentioned in Section 3. Therefore, the modification filter matrices were chosen as

$$
\left(A_{q}, B_{q}, C_{q}\right)=\left(-\lambda I_{3}, I_{3}, \lambda I_{3}\right)
$$




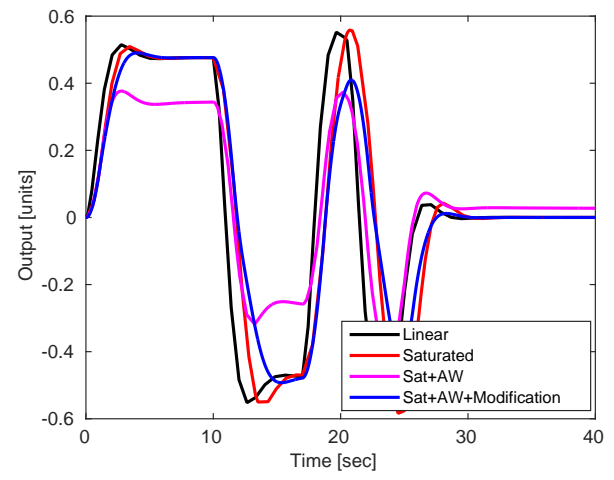

(a) Output response

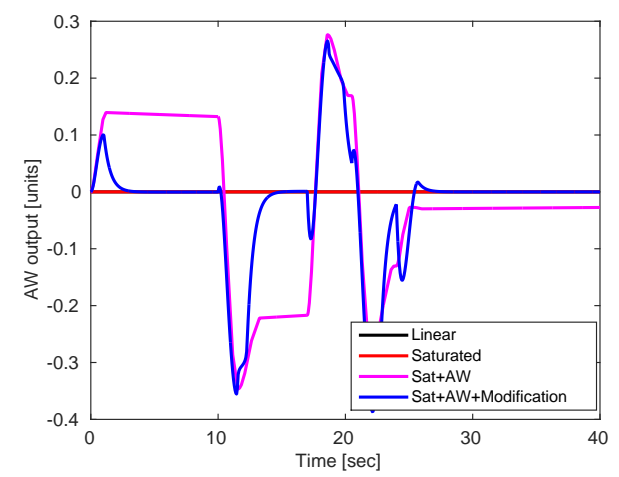

(b) $\tilde{v}$ response

Fig. 5. Lightly damped example: $y(t)$, small pulse. Nominal linear response: black. Saturated response: red. Standard anti-windup: magenta. Anti-windup with modification filter: blue. Note that the $\tilde{v}$ responses for the linear and saturated cases are zero because no anti-windup is active. Note when no modification filter is used $\tilde{v}(t) \equiv v(t)$

with $\lambda=2$. Proposition 4 was then used to verify that the system would be stable with this choice of $\mathcal{Q}$.

Figure 5 (a) shows the time history of the first output of the system due to a 3-2-1-1type reference demand of magnitude 0.5 units. The deterioration due to saturation is not catastrophic and the saturated response closely follows the linear response with a little more overshoot and a small loss of phase. However, the response with linear anti-windup is quite disappointing, with the response seeming to show a "steady state" error. This may be explained by a very slow pole being present in the anti-windup compensator, which causes one of the anti-windup signals (the second element of $\tilde{v}$ ) to decay slowly: once the anti-windup compensator has been activated, its effects linger for a long time. This is shown in Figure 5 (b): for clarity only the second element of the signals $\tilde{v}(t) \in \mathbb{R}^{3}$ have been plotted (note: when no modification filter is present $\tilde{v}(t) \equiv v(t)$ ). The blue trace in Figure 5 (a) shows the response when the modification filter is introduced: the response is much closer to the linear response, although still slightly worse than that with no anti-windup. Note that the response of the anti-windup compensator (Figure 5 (b)) now decays much faster, explaining the improved response.

Figure 6 shows the responses of system to a larger 3-2-1-1 reference demand. The pulses in this case are closer together and reference has magnitude 4 units. For this larger, faster demand Figure 6 (a) shows greater degradation in behaviour when saturation is introduced but no anti-windup is present: the output oscillates for many seconds after the last pulse is applied (at 15 seconds). When the standard antiwindup compensator is used, the response is very disappointing with the system output non-zero, and of large magnitude, 25 seconds after the last pulse demand. While not much performance can be achieved with this level of saturation, when the anti-windup modification filter is added, at least the output of the system decays to zero, as expected, within a few seconds of the last pulse being applied. As with the smaller reference demand, Figure 6 (b) explains why the standard anti-windup 


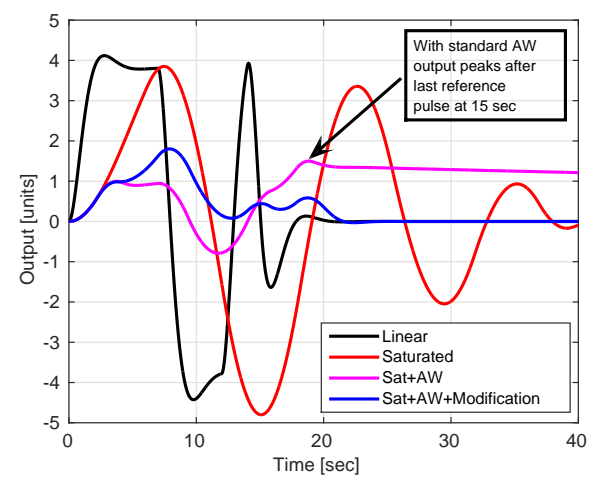

(a) Output response

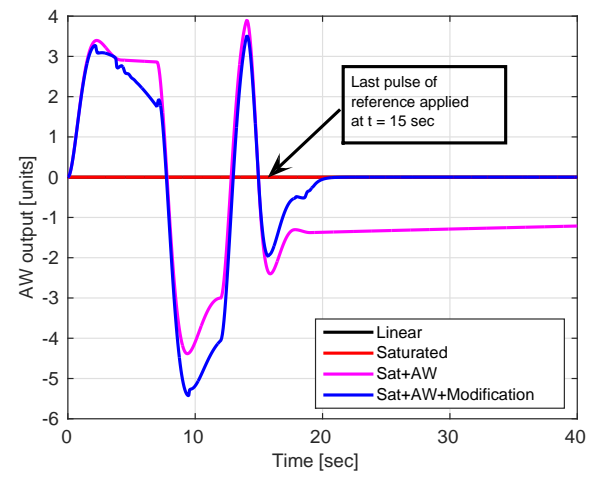

(b) $\tilde{v}$ response

Fig. 6. Lightly damped example: $y(t)$, large pulse. Nominal linear response: black. Saturated response: red. Standard anti-windup: magenta. Anti-windup with modification filter: blue. Note that the $\tilde{v}$ responses for the linear and saturated cases are zero because no anti-windup is active. Note when no modification filter is used $\tilde{v}(t) \equiv v(t)$

response is rather poor: the anti-windup signal, $v(t)$ is remains non-zero well after the the reference demand returns to zero.

\section{Conclusion}

This paper has proposed a simple nonlinear modification filter which can be appended to a linear dynamic anti-windup compensator in order, potentially, to improve performance of the overall system. The idea, roughly, is to use the modification filter to reduce the activity of the anti-windup compensator when saturation has ceased, making the dynamic compensator behave more like a static one. The work has only treated the case of a single saturation element: the multiple saturation case can be treated in a similar technical framework but in this case the number of sector conditions which will hold will increase dramatically and it is likely that some pairing of anti-windup inputs with outputs would be needed in order to obtain visibly improved performance. A direction for further improvement would be to add a robustness criterion to ensure that the use of the nonlinear modification does not impair the nominal system's robustness.

\section{Acknowledgements}

This research was supported by the the UK Engineering and Physical Sciences Research Council, Grant no. EP/N00924X/1. 


\section{A State-space matrices}

Nominal linear closed-loop system:

$$
\left[\begin{array}{c|c|c}
A_{c l} & B_{c l u} & B_{c l v} \\
\hline C_{c l} & - & D_{c l v}
\end{array}\right]=\left[\begin{array}{cc|c|c}
A_{p}+B_{p} D_{c} C_{p} & B_{p} C_{c} & -B_{p} & B_{p} D_{c v} \\
B_{c} C_{p} & A_{c} & 0 & B_{c v} \\
\hline D_{c} C_{p} & C_{c} & - & D_{c v}
\end{array}\right]
$$

State-space matrices of $\Sigma$ :

$$
\left[\begin{array}{c|c|c}
A & B_{1} & B_{2} \\
\hline C_{1} & - & - \\
\hline C_{2} & - & -
\end{array}\right]=\left[\begin{array}{ccc|c|c}
A_{c l} & B_{c l v} C_{a} & -B_{c l v} C_{q} & B_{c l u} & 0 \\
0 & A_{a} & 0 & B_{a} & 0 \\
0 & B_{q} C_{a} & A_{q} & 0 & -B_{q} \\
\hline C_{c l} & D_{c l v} C_{a} & -D_{c l v} C_{q} & - & - \\
\hline 0 & C_{a} & 0 & - & -
\end{array}\right]
$$

State-space matrices of $\Sigma_{\ell}$ :

$$
\left[\begin{array}{c|c}
A_{\ell} & B_{\ell} \\
\hline C_{\ell} & -
\end{array}\right]=\left[\begin{array}{cc|c}
A_{c l} & B_{c l v} C_{a} & B_{c l u} \\
0 & A_{a} & B_{a} \\
\hline C_{c l} & D_{c l v} C_{a} & -
\end{array}\right]
$$

\section{References}

[1] S. Tarbouriech, G. Garcia, J. M. Gomes da Silva Jr., and I. Queinnec, Stability and Stabilization of Linear Systems with Saturating Actuators. Springer, 2011.

[2] L. Zaccarian and A. R. Teel, Modern Anti-windup Synthesis: Control Augmentation for Actuator Saturation. New Jersey: Princeton University Press, 2011.

[3] P. Hippe, Windup in control. Its effects and their prevention. Germany: AIC, Springer, 2006.

[4] A. H. Glattfelder and W. Schaufelberger, Control systems with input and output constraints. Springer, London, 2003. 
[5] S. Galeani, S. Tarbouriech, M. Turner, and L. Zaccarian, "A tutorial on modern antiwindup design,” Eur J Control, vol. 15, no. 3-4, pp. 418-440, 2009.

[6] S. Tarbouriech and M. C. Turner, "Anti-windup design: an overview of some recent advances and some open problems," IET Control Theory A, vol. 3, no. 1, pp. 1-19, 2009.

[7] M. V. Kothare, P. J. Campo, M. Morari, and C. N. Nett, "A unified framework for the study of anti-windup designs," Automatica, vol. 30, no. 12, pp. 1869-1883, 1994.

[8] O. Brieger, M. Kerr, D. Leissling, I. Postlethwaite, J. Sofrony, and M. C. Turner, "Antiwindup compensation of rate saturation in an experimental aircraft," in American Control Conf., New York, 2007.

[9] O. Brieger, M. Kerr, I. Postlethwaite, M. C. Turner, and J. Sofrony, "Pilot-involvedoscillation suppression using low-order antiwindup: Flight-test evaluation," Journal of Guidance, Control, and Dynamics, vol. 35, no. 2, pp. 471-483, 2012.

[10] L. Zaccarian and A. R. Teel, "Nonlinear scheduled anti-windup design for linear systems," IEEE T Automat Contr, vol. 49, no. 11, pp. 2055-2061, 2004.

[11] M. C. Turner, G. Herrmann, and I. Postlethwaite, "Improving local anti-windup performance: Preliminary results on a two-stage approach," in IFAC P World Congr, Prague, 2005.

[12] S. Galeani, A. R. Teel, and L. Zaccarian, "Constructive nonlinear anti-windup design for exponentially unstable linear plants," Syst Control Lett, vol. 56, no. 5, pp. 357-365, 2007.

[13] S. Sajjadi-Kia and F. Jabbari, "Modified anti-windup compensators for stable plants," IEEE T Automat Contr, vol. 54, no. 8, pp. 1934-1939, 2009.

[14] J. Sofrony and M. C. Turner, "A simple scheduled anti-windup design," in American Control Conf., Portland, 2014.

[15] X. Wu and Z. Lin, "Dynamic anti-windup design in anticipation of actuator saturation," Int J Robust Nonlin, vol. 24, no. 2, pp. 295-312, 2014.

[16] M. Bruckner, S. Galeani, and L. Del Re, "Robustified anti-windup via switching adaptation," IEEE Transactions on Automatic Control, vol. 58, no. 3, pp. 731-737, 2013.

[17] S. Galeani, S. Onuri, and L. Zaccarian, "Nonlinear scheduled control for linear systems subject to saturation with application to anti-windup control," in IEEE Decis Contr P, New Orleans, 2007, pp. 1168-1173.

[18] M. C. Turner, G. Herrmann, and I. Postlethwaite, "Incorporating robustness requirements into anti-windup design," IEEE T Automat Contr, vol. 52, no. 10, 2007.

[19] M. C. Turner and I. Postlethwaite, "A new perspective on static and low order antiwindup synthesis," Int J Control, vol. 77, no. 1, pp. 27-44, 2004. 
[20] J.-M. Biannic, C. Roos, and S. Tarbouriech, "A practical method for fixed-order antiwindup design," in $7^{\text {th }}$ IFAC Symposium on Nonlinear Control Systems (NOLCOS), Pretoria, South Africa, 2007.

[21] M. C. Turner and G. Herrmann, "A non-square sector condition and its application in deferred-action anti-windup compensator design," Automatica, vol. 50, no. 1, pp. 268-276, 2014.

[22] H. Khalil, Nonlinear Systems. New Jersey: Prentice Hall, 1996.

[23] P. Park, "Stability criteria of sector and slope restricted Lur'e systems," IEEE Transactions on Automatic Control, vol. 47, no. 2, pp. 308-313, 2002.

[24] M. C. Turner and M. Kerr, "Lyapunov functions and $\mathcal{L}_{2}$ gain bounds for systems with slope restricted nonlinearities," Systems \& Control Letters, vol. 69, pp. 1-6, 2014.

[25] G. Valmorbida, R. Drummond, and S. Duncan, "Positivity conditions of Lyapunov functions for systems with slope restricted nonlinearities," in American Control Conference. American Automatic Control Council (AACC), 2016, pp. 258-263. 